Groups Geom. Dyn. 8 (2014), 143-155

DOI $10.4171 / \mathrm{GGD} / 220$
Groups, Geometry, and Dynamics

(C) European Mathematical Society

\title{
Rank gradient in co-final towers of certain Kleinian groups
}

\author{
Darlan Girão
}

\begin{abstract}
We prove that if the fundamental group of an orientable finite volume hyperbolic 3 -manifold has finite index in the reflection group of a right-angled ideal polyhedron in $\mathbb{H}^{3}$ then it has a co-final tower of finite sheeted covers with positive rank gradient. The manifolds we consider are also known to have co-final towers of covers with zero rank gradient.
\end{abstract}

Mathematics Subject Classification (2010). 57M05, 57M10, 57M50.

Keywords. Kleinian groups, rank of groups.

\section{Introduction}

Let $G$ be a finitely generated group. The rank of $G$ is the minimal cardinality of a generating set, and is denoted by $\operatorname{rk}(G)$. If $G_{j}$ is a finite index subgroup of $G$, the Reidemeister-Schreier process ([LS]) gives an upper bound on the rank of $G_{j}$ :

$$
\operatorname{rk}\left(G_{j}\right)-1 \leq\left[G: G_{j}\right](\operatorname{rk}(G)-1) .
$$

Recently Lackenby introduced the notion of rank gradient ([La1]). Given a finitely generated group $G$ and a collection $\left\{G_{j}\right\}$ of finite index subgroups, the rank gradient of the pair $\left(G,\left\{G_{j}\right\}\right)$ is defined by

$$
\operatorname{rgr}\left(G,\left\{G_{j}\right\}\right)=\lim _{j \rightarrow \infty} \frac{\operatorname{rk}\left(G_{j}\right)-1}{\left[G: G_{j}\right]}
$$

We say that the collection of finite index subgroups $\left\{G_{j}\right\}$ is co-final if $\bigcap_{j} G_{j}=\{1\}$, and we call it a tower if $G_{j+1}<G_{j}$.

In some particular cases it is easy to determine rank gradient, for example:

(1) When $G$ is a free group, the rank gradient of any pair $\left(G,\left\{G_{j}\right\}\right)$ is positive.

(2) The same is true if $G$ is the fundamental group of a closed surface $S$ with $\chi(S)<0$;

(3) If $G \rightarrow F_{2}$, where $F_{2}$ is the free group on two generators then, using (1), one can find a tower (not co-final) of subgroups with positive rank gradient; 
(4) If $G$ is virtually abelian or if $G$ is the fundamental group of a virtually fibered 3-manifold then there are towers with zero rank gradient. In the latter case we consider the subgroups coming from the cyclic covers of the fibered manifold.

(5) $\operatorname{SL}(n, \mathbb{Z}), n>2$, has zero rank gradient with respect to towers of congruence subgroups ([Ti], [La1]).

However, determining the rank gradient of a co-final tower is very hard in general. For example, the following question is the motivation for this note:

Question 1. Does there exist a torsion free finite covolume Kleinian group $G$ with a co-final tower $\left\{G_{j}\right\}$ such that $\operatorname{rgr}\left(G,\left\{G_{j}\right\}\right)>0$ ?

The main result of this note provides infinitely many such examples. To state it we introduce some notation.

If $M_{1}$ is an orientable finite volume hyperbolic 3-manifold, we call the family of covers $\left\{M_{j} \rightarrow M_{1}\right\}$ co-final (resp. a tower) if $\left\{\pi_{1}\left(M_{j}\right)\right\}$ is co-final (resp. a tower). By rank gradient of the pair $\left(M_{1},\left\{M_{j}\right\}\right), \operatorname{rgr}\left(M_{1},\left\{M_{j}\right\}\right)$, we mean the rank gradient of $\left(\pi_{1}\left(M_{1}\right),\left\{\pi_{1}\left(M_{j}\right)\right\}\right)$.

Theorem 3.1. Let $M_{1}$ be an orientable finite volume hyperbolic 3-manifold whose fundamental group has finite index in the reflection group of a totally geodesic rightangled ideal polyhedron $P_{1}$ in $\mathbb{H}^{3}$. Then there exists a co-final tower of finite sheeted covers $\left\{M_{j} \rightarrow M\right\}$ with positive rank gradient.

This theorem relates to the work of Abért and Nikolov ([AN]), and in particular to a question about cost of group actions ([Ga]).

Question 2. Let $G$ be finitely generated and $\left\{G_{j}\right\}$ be a co-final tower of normal subgroups of $G$. Does $\operatorname{rgr}\left(G,\left\{G_{j}\right\}\right)$ depend on the tower $\left\{G_{j}\right\}$ ?

We remark that the manifolds in Theorem 3.1 are known to have towers of covers with zero rank gradient. Agol proved in [Ag] that if the fundamental group of a 3dimensional manifold satisfies an algebraic condition, called RFRS, then it virtually fibers. He also proved in [Ag] that the manifolds of the type considered in Theorem 3.1 are virtually RFRS. Therefore, given $M_{1}$ as in Theorem 3.1, it is possible to find a tower $\left\{\Gamma_{j}\right\}$ with $\operatorname{rgr}\left(\pi_{1}\left(M_{1}\right),\left\{\Gamma_{j}\right\}\right)=0$.

The main idea of the proof of Theorem 3.1 is as follows: given $P_{1}$ as in the theorem, construct a collection of polyhedra $\left\{P_{j}\right\}$ whose reflection groups have finite index $2^{j-1}$ in the reflection group of $P_{1}$. If one is given an orientable hyperbolic 3-manifold $M_{1}$ whose fundamental group has finite index in the reflection group of $P_{1}$ then $M_{1}$ has at least as many cusps as the number of vertices of $P_{1}$. We may find manifold covers $M_{j} \rightarrow M_{1}$ so that $M_{j}$ is a $2^{j-1}$-sheeted covering and has at least as many cusps as the number of ideal vertices of $P_{j}$. We then show that the $P_{j}$ can be chosen so that the number of its vertices is of the same magnitude as $2^{j}$. 
The paper will be organized as follows: Section 2 sets up notation and we recall a characterization of right-angled ideal polyhedra using Andreev's theorem ([An]). We then show how the construction of the family $\left\{P_{j}\right\}$ will be done. In Section 3 we prove Theorem 3.1. Section 4 contains all the technical results we need to estimate $\operatorname{rk}\left(\pi_{1}\left(M_{j}\right)\right)$. In Section 5 we show how to construct $\left\{P_{j}\right\}$ so that the family $\left\{M_{j}\right\}$ is co-final. The idea for this appears in $[\mathrm{Ag}]$ (Theorem 2.2) and we include a proof here for completeness. Section 6 contains some final remarks and further questions.

Ackowledgements. I am very grateful to my thesis advisor, Alan Reid, for his extraordinary guidance and unwavering support. I am also thankful to Ian Agol, Grant Lakeland and Mark Norfleet for helpful conversations. The author was partially supported by CAPES/Fulbright Grant BEX 2411/05-9.

\section{Set up}

An abstract polyhedron $\mathcal{P}_{1}$ is a cell complex on $S^{2}$ which can be realized by a convex Euclidean polyhedron. A labeling of $\mathcal{P}_{1}$ is a map

$$
\Theta: \operatorname{Edges}\left(\mathscr{P}_{1}\right) \rightarrow(0, \pi / 2] .
$$

The pair $\left(\mathscr{P}_{1}, \Theta\right)$ is a labeled abstract polyhedron. A labeled abstract polyhedron is said to be realizable as a hyperbolic polyhedron if there exists a hyperbolic polyhedron $P_{1}$ such that there is a label preserving graph isomorphism between the 1-skeleton of $P_{1}$ with edges labeled by dihedral angles and the 1 -skeleton of $\mathcal{P}_{1}$ with edges labeled by $\Theta$.

Let $P_{1}$ be a totally geodesic right-angled ideal polyhedron in $\mathbb{H}^{3}$ (that is, faces of $P_{1}$ are contained in hyperplanes and all vertices of $P_{1}$ lie in the boundary at infinity $S_{\infty}^{2}$, where we here we consider the ball model for $\mathbb{H}^{3}$ ). We consider the 1-skeleton of $P_{1}$ as a graph $\Gamma_{1} \subset S^{2}$ with labels $\theta_{e}=\pi / 2$. Let $\Gamma_{1}^{*}$ be its dual graph. A $k$-circuit is a simple closed curve composed of $k$ edges in $\Gamma_{1}^{*}$. A prismatic $k$-circuit is a $k$-circuit $\gamma$ so that no two edges of $\Gamma_{1}$ which correspond to edges traversed by $\gamma$ share a vertex. Andreev's theorem for right-angled ideal polyhedra in $\mathbb{H}^{3}$ ([An], see also $[\mathrm{At}])$ can be stated as:

Theorem 2.1. Let $\mathcal{P}_{1}$ be an abstract polyhedron. Then $\mathcal{P}_{1}$ is realizable as a rightangled ideal polyhedron $P_{1}$ if and only if

(1) $P_{1}$ has at least 6 faces;

(2) vertices have valence 4;

(3) for any triple of faces of $P_{1},\left(f_{i}, f_{j}, f_{k}\right)$, such that $f_{i} \cap f_{j}$ and $f_{j} \cap f_{k}$ are edges of $P_{1}$ with distinct endpoints, $f_{i} \cap f_{k}=\emptyset$;

(4) there are no prismatic 4-circuits. 
The above theorem implies that the 1-skeleton of $P_{1}$ is a 4-valent graph. The faces can therefore be checkerboard colored. Reflecting $P_{1}$ along a face $f_{1}$ gives a polyhedron $P_{2}$ which is also right-angled, ideal and totally geodesic with checkerboard colored faces (see figure below). We construct a sequence of polyhedra $P_{1}, P_{2}, \ldots, P_{j}, \ldots$ recursively, whereby $P_{j+1}$ is obtained from $P_{j}$ by reflection along a face $f_{j}$. The faces of $P_{j+1}$ are colored accordingly with the coloring of the faces of $P_{j}$.

The notation for the remainder of the paper is as follows: the number of vertices in the face $f_{j}$ is denoted by $S_{f_{j}}$ and $\phi_{f_{j}}$ denotes the reflection along $f_{j} . B_{j}$ and $W_{j}$ represent the maximal number of ideal vertices on a black or white face of the polyhedron $P_{j}$, respectively. $V_{j}$ denotes the total number of vertices on $P_{j}$.

Throughout, the construction of the polyhedra $P_{j}$ will be done in an alternating fashion with respect to the color of the faces: $P_{2 j}$ is obtained from $P_{2 j-1}$ by refection along a black face and $P_{2 j+1}$ is obtained from $P_{2 j}$ by reflection along a white face.
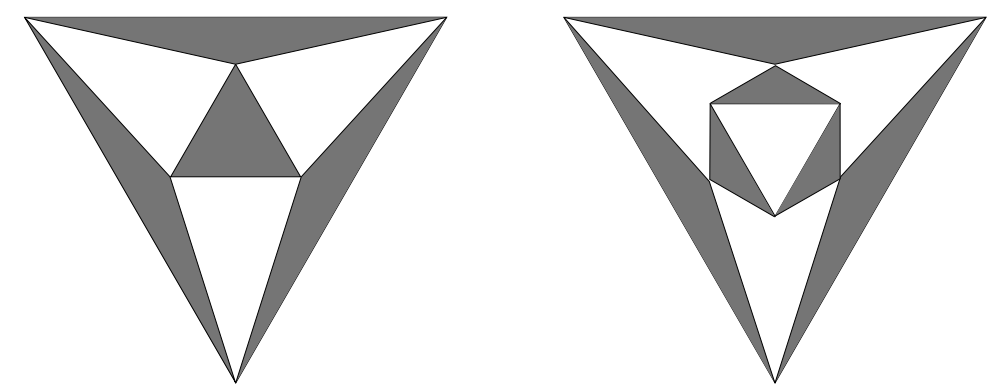

Figure 1. Polyhedron $P_{1}$ reflected along central black face yields $P_{2}$.

\section{Main theorem}

In this section we prove:

Theorem 3.1. Let $M_{1}$ be an orientable finite volume hyperbolic 3-manifold whose fundamental group has finite index in the reflection group of a right-angled ideal polyhedron $P_{1}$ in $\mathbb{H}^{3}$. Then there exists a co-final tower of finite sheeted covers $\left\{M_{j} \rightarrow M\right\}$ with positive rank gradient.

Our construction of the family $\left\{M_{j}\right\}$ was inspired by the proof of Theorem 2.2 of Agol's paper ([Ag]). The proof that this family can be made co-final is given in Section 5 (following $[\mathrm{Ag}]$ ).

Proof of Theorem 3.1. Consider the family of polyhedra $\left\{P_{j}\right\}$ obtained from $P_{1}$ as described above. Denote by $G_{j}$ the reflection group of $P_{j}$ and observe that $G_{j+1}$ 
is a subgroup of $G_{j}$ of index 2. $G_{1}$ acts on $\mathbb{H}^{3}$ with fundamental domain $P_{1}$. The orbifold $\mathbb{H}^{3} / G_{1}$ is non-orientable, and may be viewed as $P_{1}$ with its faces mirrored. The singular locus is the 2-skeleton of $P_{1}$. Each ideal vertex of $P_{1}$ corresponds to a cusp of $\mathbb{H}^{3} / G_{1}$.

Let $M_{1}$ be an orientable cusped hyperbolic 3-manifold such that $\pi_{1}\left(M_{1}\right)$ has finite index in $G_{1}$. Let $M_{j} \rightarrow M_{1}$ be the cover of $M_{1}$ whose fundamental group is $\pi_{1}\left(M_{j}\right)=\pi_{1}\left(M_{1}\right) \cap G_{j}$. Since $\left[G_{j}: G_{j+1}\right]=2$, we must have $\left[\pi_{1}\left(M_{j}\right)\right.$ : $\left.\pi_{1}\left(M_{j+1}\right)\right] \leq 2$. Also note that since $\operatorname{vol}\left(P_{j}\right)=2^{j-1} \operatorname{vol}\left(P_{1}\right)$, for all but finitely many $j$ (at most $\left[G_{1}: \pi_{1}\left(M_{1}\right)\right]$ ) we must have $\left[\pi_{1}\left(M_{j}\right): \pi_{1}\left(M_{j+1}\right)\right]=2$. We may thus assume that $\left[\pi_{1}\left(M_{j}\right): \pi_{1}\left(M_{j+1}\right)\right]=2$. By mirroring the faces of $P_{j}$, it may be regarded as a non-orientable finite volume orbifold (as described before). This implies that $M_{j} \rightarrow P_{j}$ is an orientable finite sheeted cover for $j=1,2, \ldots$

Note that $\left[\pi_{1}\left(M_{1}\right): \pi_{1}\left(M_{j}\right)\right]=2^{j-1}$. Thus to show that the family $\left\{M_{j} \rightarrow M_{1}\right\}$ has positive rank gradient we will establish that $\operatorname{rk}\left(\pi_{1}\left(M_{j}\right)\right)$ grows with the same magnitude as $2^{j}$.

By "half lives half dies", an easy lower bound on the rank of the fundamental group of an orientable finite volume hyperbolic 3-manifold is the number of its cusps. Since the cusps of $P_{j}$ correspond to its ideal vertices and the number of cusps does not go down under finite sheeted covers, it must be that $M_{j}$ has at least as many cusps as the number of ideal vertices of $P_{j}$.

Recall that $B_{j}$ and $W_{j}$ are the maximal number of ideal vertices on a black or white face of the polyhedron $P_{j}$, respectively, and $V_{j}$ is the total number of vertices on $P_{j}$. The claims below (proved in Section 4) give us the estimates we need for $V_{j}$ in terms of $V_{1}, B_{1}$ and $W_{1}$.

Claim 1. $V_{1} \geq B_{1}+W_{1}-1$.

Claim 2. For any $j \geq 6, V_{j} \geq 2^{j-1} V_{1}-2^{j-1}\left(B_{1}+W_{1}\right)+2^{j-1}+2^{j-2}$.

Given these, we argue as follows:

$$
\begin{aligned}
\operatorname{rgr}\left(M_{1},\left\{M_{j}\right\}\right) & =\lim _{j \rightarrow \infty} \frac{\operatorname{rk}\left(\pi_{1}\left(M_{j}\right)\right)-1}{\left[\pi_{1}\left(M_{1}\right): \pi_{1}\left(M_{j}\right)\right]} \\
& \geq \lim _{j \rightarrow \infty} \frac{V_{j}-1}{2^{j-1}} \\
& \geq \lim _{j \rightarrow \infty} \frac{2^{j-1} V_{1}-2^{j-1}\left(B_{1}+W_{1}\right)+2^{j-1}+2^{j-2}-1}{2^{j-1}} \\
& \geq \lim _{j \rightarrow \infty} \frac{2^{j-1}\left(B_{1}+W_{1}-1\right)-2^{j-1}\left(B_{1}+W_{1}\right)+2^{j-1}+2^{j-2}-1}{2^{j-1}} \\
& \geq \lim _{j \rightarrow \infty} \frac{2^{j-2}-1}{2^{j-1}}=\frac{1}{2}
\end{aligned}
$$

which proves the theorem. 


\section{Lower bounds on number of ideal vertices of $\boldsymbol{P}_{\boldsymbol{j}}$}

We now proceed to prove Claims 1 and 2. This requires several preliminary results.

Lemma 4.1. Let $P_{j+1}$ be obtained from $P_{j}$ by reflection along a face $f_{j}$. Then $V_{j+1}=2 V_{j}-S_{f_{j}}$.

Proof. Here we abuse notation and write $v \in f_{j}$ if $v$ is an ideal vertex of the face $f_{j}$ and write $v \notin f_{j}$ otherwise. Note that if $v \notin f_{j}$, then $v$ yields two vertices on $P_{j+1}$, namely, $v$ and $\phi_{f_{j}}(v)$. If $v \in f_{j}$, then it yields a single vertex ( $v$ itself).

If $v \notin f_{j}$, then, by the observation above, $v$ yields two ideal vertices on $P_{j+1}$. Since a total of $S_{f_{j}}$ ideal vertices lie in $f_{j}$ and $V_{j}-S_{f_{j}}$ do not, it must be that that

$$
V_{j+1}=2\left(V_{j}-S_{f_{j}}\right)+S_{f_{j}}=2 V_{j}-S_{f_{j}} .
$$

Recall also that the construction of the family of polyhedra $\left\{P_{j}\right\}$ is made in an alternating fashion with respect to the color of the faces: $P_{2 j}$ is obtained from $P_{2 j-1}$ by refection along a black face and $P_{2 j+1}$ is obtained from $P_{2 j}$ by reflection along a white face.

Corollary 4.2. For $j \geq 1$,

(1) $V_{2 j} \geq 2 V_{2 j-1}-B_{2 j-1}$, and

(2) $V_{2 j+1} \geq 2 V_{2 j}-W_{2 j}$.

Proof. $P_{2 j}$ is obtained from $P_{2 j-1}$ by refection along a black face $f_{2 j-1}$, thus $S_{f_{2 j-1}} \leq B_{2 j-1}$. By the lemma, $V_{2 j}=2 V_{2 j-1}-S_{f_{2 j-1}}$ and therefore $V_{2 j} \geq$ $2 V_{2 j-1}-B_{2 j-1}$. The second inequality is similar.

With the notation established above we now find lower bounds for the $V_{j}$ in terms of $V_{1}, B_{1}$ and $W_{1}$. First we need to find upper bounds for $B_{j}$ and $W_{j}$ in terms of $B_{1}$ and $W_{1}$. To do this in a way that will fit our purposes we establish two properties of the family $\left\{P_{j}\right\}$. As before, denote by $\phi_{f_{j}}$ the reflection along the face $f_{j}$.

Lemma 4.3. (1) If $P_{j}$ is reflected along a white (resp. black) face $f_{j}$, all black faces $f_{*}$ adjacent to $f_{j}$ yield new black faces $\tilde{f}_{*}$ on $P_{j+1}$. The number $S_{\tilde{f}_{*}}$ of ideal vertices on $\tilde{f}_{*}$ is $2 S_{f_{*}}-2$.

(2) A face $f_{*}$ not adjacent to $f_{j}$ yield two new faces, $f_{*}$ itself and $\phi_{f}\left(f_{*}\right)$, both with $S_{f_{*}}$ vertices.

Proof. For the first property, reflecting $f_{*}$ along $f_{j}$ gives a face $\phi_{f_{j}}\left(f_{*}\right)$ in $P_{j+1}$ adjacent to $f_{*}$. The dihedral angle between $f_{*}$ and $\phi_{f}\left(f_{*}\right)$ is $\pi$. Thus, on $P_{j+1}$, they correspond to a single face denoted by $\tilde{f}_{*}$. The number of ideal vertices on $\tilde{f}_{*}$ is exactly $2 S_{f_{*}}-2$. The second property should be clear. See figure 1 for an illustration of these properties. 
As an immediate consequence we have

\section{Corollary 4.4.}

(1) $\left\{\begin{array}{l}B_{2 j}=B_{2 j-1}, \\ W_{2 j} \leq 2 W_{2 j-1}-2 .\end{array}\right.$
(2) $\left\{\begin{array}{l}B_{2 j+1} \leq 2 B_{2 j}-2, \\ W_{2 j+1}=W_{2 j} .\end{array}\right.$

We are now in position to estimate the values $B_{j}$ and $W_{j}$ in terms of $B_{1}$ and $W_{1}$.

Theorem 4.5. With the notation as before we have

(1) $W_{2 j+1}=W_{2 j} \leq 2^{j} W_{1}-\sum_{l=1}^{j} 2^{l}$, and

(2) $B_{2 j+2}=B_{2 j+1} \leq 2^{j} B_{1}-\sum_{l=1}^{j} 2^{l}$.

Proof. We proceed by induction. By Corollary 4.4 these statements are true for $j=$ 1. Suppose they are also true for $j \leq n$. We now want to estimate $B_{2 n+3}=B_{2 n+4}$ and $W_{2 n+2}=W_{2 n+3}$. The hypothesis is that

$$
W_{2 j+1}=W_{2 j} \leq 2^{n} W_{1}-\sum_{l=1}^{n} 2^{l}
$$

and

$$
B_{2 n+2}=B_{2 n+1} \leq 2^{n} B_{1}-\sum_{l=1}^{n} 2^{l}
$$

$P_{2 n+2}$ is obtained from $P_{2 n+1}$ by reflection along a black face, denoted by $f$. White faces on $P_{2 n+1}$ adjacent to $f$ yield new white faces on $P_{2 n+2}$ with at most $2 W_{2 n+1}-2$ vertices, by Corollary 4.4. But

$$
2 W_{2 n+1}-2 \leq 2\left[2^{n} W_{1}-\sum_{l=1}^{n} 2^{l}\right]-2=2^{(n+1)} W_{1}-\sum_{l=1}^{n+1} 2^{l}
$$

which gives the desired result for $W_{2 n+2}$ and $W_{2 n+3}$. Finally, $P_{2 n+3}$ is obtained from $P_{2 n+2}$ by a reflection along a white face, again denoted by $f$. Since black faces of $P_{2 n+2}$ have at most $B_{2 n+2}\left(=B_{2 n+1}\right)$ vertices, black faces of $P_{2 n+3}$ will have at most $2 B_{2 n+1}-2$ vertices, again by Corollary 4.4 . But

$$
2 B_{2 n+1}-2 \leq 2\left[2^{n} B_{1}-\sum_{l=1}^{n} 2^{l}\right]-2=2^{(n+1)} B_{1}-\sum_{l=1}^{n+1} 2^{l}
$$

This establishes the result for $B_{2 n+3}$ and $B_{2 n+4}$. 
Theorem 4.6. With the notation as before, and for $j \geq 3$,

(1) $V_{2 j} \geq 2^{2 j-1} V_{1}-B_{1} \sum_{l=j-1}^{2 j-2} 2^{l}-W_{1} \sum_{l=j}^{2 j-2} 2^{l}+\sum_{l=j+2}^{2 j-1} 2^{l}+2^{j}+2$, and

(2) $V_{2 j+1} \geq 2^{2 j} V_{1}-B_{1} \sum_{l=j}^{2 j-1} 2^{l}-W_{1} \sum_{l=j}^{2 j-1} 2^{l}+\sum_{l=j+2}^{2 j} 2^{l}+2$.

Proof. Lower bounds estimates for $V_{1}, \ldots, V_{7}$ are found recursively. $V_{1}, V_{2}, V_{3}, V_{4}$ and $V_{5}$ do not fit these formulas but $V_{6}$ and $V_{7}$ do. The statement is then true for $j=3$. We now proceed by induction, using the previous proposition and Corollary 4.2. Suppose it is true for $j \leq n, n \geq 3$. We want to show this holds true for $j=n+1$. By Corollary $4.2, V_{2 n+2} \geq 2 V_{2 n+1}-B_{2 n+1}$. The hypothesis is that

$$
V_{2 n+1} \geq 2^{2 n} V_{1}-B_{1} \sum_{l=n}^{2 n-1} 2^{l}-W_{1} \sum_{l=n}^{2 n-1} 2^{l}+\sum_{l=n+2}^{2 n} 2^{l}+2 .
$$

We also know that

$$
B_{2 n+1} \leq 2^{n} B_{1}-\sum_{l=1}^{n} 2^{l}
$$

Thus

$$
\begin{aligned}
V_{2 n+2} & \geq 2 V_{2 n+1}-B_{2 n+1} \\
& \geq 2\left[2^{2 n} V_{1}-B_{1} \sum_{l=n}^{2 n-1} 2^{l}-W_{1} \sum_{l=n}^{2 n-1} 2^{l}+\sum_{l=n+2}^{2 n} 2^{l}+2\right]-\left[2^{n} B_{1}-\sum_{l=1}^{n} 2^{l}\right] \\
& =2^{2 n+1} V_{1}-B_{1} \sum_{l=n}^{2 n-1} 2^{l+1}-W_{1} \sum_{l=n}^{2 n-1} 2^{l+1}+\sum_{l=n+2}^{2 n} 2^{l+1}+2^{2}+\sum_{l=1}^{n} 2^{l} \\
& =2^{2 n+1} V_{1}-B_{1} \sum_{l=n}^{2 n} 2^{l}-W_{1} \sum_{l=n+1}^{2 n} 2^{l}+\sum_{l=n+3}^{2 n+1} 2^{l}+2^{n+1}+2
\end{aligned}
$$

which establishes $(1)$ for $2(n+1)=2 n+2$.

We use the exact same idea and the estimate for $V_{2 n+2}$ to establish (2) for $2(n+1)+1=2 n+3$.

Corollary 4.7. For any $j \geq 6$,

$$
V_{j} \geq 2^{j-1} V_{1}-2^{j-1}\left(B_{1}+W_{1}\right)+2^{j-1}+2^{j-2} .
$$

Hence Claim 2 in the proof of Theorem 3.1 is proved. We now prove

Claim 1. $V_{1} \geq B_{1}+W_{1}-1$. 
Proof. Let $f_{b}$ and $f_{w}$ be black and white faces of $P_{1}$ with maximal number of vertices, i.e., $S_{f_{b}}=B_{1}$ and $S_{f_{w}}=W_{1}$.

Case 1: The faces $f_{b}$ and $f_{w}$ are not adjacent. Here we get $V_{1} \geq B_{1}+W_{1}$ and the claim follows.

Case 2: The faces $f_{b}$ and $f_{w}$ are adjacent. Since $f_{b}$ and $f_{w}$ share exactly 2 vertices we see that $V_{1} \geq B_{1}+W_{1}-2$. Suppose we have equality. Then every vertex of $P_{1}$ must be a vertex of either $f_{b}$ or $f_{w}$. Recall that we can visualize the 1 -skeleton of $P_{1}$ as lying in $S^{2}$. Label the vertices of $P_{1}$ by $\left\{v_{1}, \ldots, v_{k}\right\}$. The assumption is that all these vertices lie in the boundary of the disk $D=\overline{\left(f_{b} \cup f_{w}\right)} \subset S^{2}$. By Andreev's theorem, $P_{1}$ has at least 6 faces, every face is at least 3 -sided and all vertices are 4-valent. Denoting by $F_{1}$ and $E_{1}$ the number of faces and edges of $P_{1}$ respectively we have the relation $V_{1}-E_{1}+F_{1}=2$. Since vertices are 4-valent we also have $E_{1}=2 V_{1}$. From these relations and $F_{1} \geq 6$, we get $V_{1} \geq 4$. At two of the vertices, say $v_{1}$ and $v_{2}$, three of the emanating edges lie in $D$ and one does not. Denote the ones that do not lie in $D$ by $e_{1}$ and $e_{2}$, respectively. At all other $v_{i}$ we have two edges that lie in $D$ and two that do not. Denote the latter by $e_{i}, e_{i}^{\prime}$. We have a total of $2(k-2)+2=2 k-2$ edges not in $D$. The problem we have now is combinatorial:

Given the disk $D^{\prime}=\overline{S^{2}-D}$ and the points $v_{1}, \ldots, v_{k} \in \partial D^{\prime}, k \geq 4$, is it possible to subdivide $D^{\prime}$ by $2 k-2$ edges in a way that exactly one edge emanates from both $v_{1}$ and $v_{2}$ and exactly two edges emanate from $v_{3}, \ldots, v_{k}$ in such a way that no pair of edges intersect and every face on the subdivision of $D^{\prime}$ is at least 3-sided (here we also consider sides coming from the boundary)?

A simple argument will show that the answer to this question is negative. Orient the boundary of $D^{\prime}$ counterclockwise. Starting at $v_{1}$, draw the edge $e_{1}$ emanating from it. The other endpoint of $e_{1}$ is some vertex $v_{i_{1}}$. Consider the vertices contained in the segment $\left[v_{1}, v_{i_{1}}\right] \subset \partial D^{\prime}$ in the given orientation. If there are no vertices at all, then we must have a 2 -sided face, which is not possible. Therefore, by relabeling, we may assume $v_{2}$ is the first vertex between $v_{1}$ and $v_{i_{1}}$. Observe that the edges emanating from $v_{2}$ are trapped between the edge $e_{1}$ and $\partial D^{\prime}$. Draw an edge $e_{2}$ emanating from $v_{2}$ with the second endpoint $v_{i_{2}}$. It must be that $v_{i_{2}}$ also lies in $\left[v_{1}, v_{i_{1}}\right]$, or else we find a pair of intersecting edges. As above, there must be a vertex in the segment $\left[v_{2}, v_{i 2}\right]$. By repeating the above argument eventually we find a 2-sided face, which is not possible. Therefore it must be that $V_{1}>B_{1}+W_{1}-2$.

\section{Co-finalness}

In this section we provide a way of choosing the black or white faces on the polyhedra $P_{j}$ along which it is reflected in such a way that the resulting family $\left\{M_{j}\right\}$ of manifolds is co-final. The main result of this section, Theorem 5.1, appears as part of the proof 
of Theorem 2.2 of [Ag]. We include a proof here for completeness. To better describe this construction we need to change notation slightly by adding another index.

Start with $P_{1}$ and relabel it $P_{11}$. Reflect along a black face $f_{11}$ obtaining $P_{12}$. Let $\phi_{f_{11}}$ represent such reflection. Observe that if $f$ is adjacent to $f_{11}$, then $f \cup \phi_{f_{11}}(f)$ corresponds to a single face on $P_{12}$. We call $f$ and $\phi_{f_{11}}(f)$ subfaces of $f \cup \phi_{f_{11}}(f)$. Next reflect $P_{12}$ along a white face $f_{12}$, which is also a face of $P_{11}$ or contains a face of $P_{11}$ as a subface, obtaining $P_{13}$. We construct a subcollection $P_{11}, \ldots, P_{1 k_{1}}$ of polyhedra such that

(i) If $P_{1 j}$ is obtained from $P_{1(j-1)}$ by reflection along a white (black) face then $P_{1(j+1)}$ is obtained from $P_{1 j}$ by reflection along a black (white) face.

(ii) Whenever possible, the face $f_{1 j}$ must be a face of $P_{11}$ or contain a face of $P_{11}$ as a subface.

(iii) No faces of $P_{11}$ are subfaces of $P_{1 k_{1}}$.

Now set $P_{1 k_{1}}:=P_{21}$.

Suppose $P_{n 1}$ has been constructed. Construct the subcollection of polyhedra $P_{n 1}, \ldots, P_{n k_{n}}$ such that

(i) The reflections were performed in a alternating fashion with respect to the color of the faces;

(ii) Whenever possible, the face $f_{n j}$ must be a face of $P_{n 1}$ or contain a face of $P_{n 1}$ as a subface.

(iii) No faces of $P_{n 1}$ are subfaces of $P_{n k_{n}}$.

Now set $P_{n k_{n}}:=P_{(n+1) 1}$. Inductively we obtain a collection of polyhedra

$$
P_{11}, P_{12}, \ldots, P_{1 k_{1}}:=P_{21}, \ldots, P_{2 k_{2}}:=P_{31}, \ldots, P_{n k_{n}}:=P_{(n+1) 1}, \ldots
$$

satisfying (i), (ii) and (iii) above.

Let $G_{i j}$ be the reflection group of $P_{i j}$ and let $M_{i j}$ be the cover of $M_{11}$ whose fundamental group is $\pi_{1}\left(M_{i j}\right)=\pi_{1}\left(M_{11}\right) \cap G_{i j}$. Co-finalness of the family $\left\{M_{i j} \rightarrow\right.$ $\left.M_{11}\right\}$ is an immediate consequence of

Theorem 5.1. Let $G_{i j}$ be as above. Then $\bigcap_{i j} G_{i j}=\{1\}$.

In order to prove this theorem we consider the base point for the fundamental group of each $P_{i j}$ (viewed as orbifolds with their faces mirrored) to be the barycenter $x_{0}$ of $P_{11}$.

Proof of Theorem 5.1. Set $R_{i j}=\inf _{\gamma}\{\ell(\gamma)\}$, where $\gamma$ is an arc with endpoints in faces (possibly edges) of $P_{i j}$ going through $x_{0}$. Note that, by construction, $\lim _{i \rightarrow \infty} R_{i j}=\infty$. For a non-trivial element $g \in G_{11}$ set $R_{g}=\inf _{[\alpha]=g}\{\ell(\alpha)\}$, where $\alpha$ is a loop in $P_{11}$ based at $x_{0}$ and $[\alpha]$ represents its homotopy class. Let $\alpha_{g}$ be a loop in $P_{11}$ based at $x_{0}$ such that $\left[\alpha_{g}\right]=g$ and $\ell\left(\alpha_{g}\right) \leq R_{g}+1$. 
We claim that for sufficiently large $i$ one cannot have $g \in G_{i j}$. In fact, if $\alpha_{i j}$ is any nontrivial loop in $P_{i j}$ based at $x_{0}$, then this loop bounces off faces of $P_{i j}$, yielding an $\operatorname{arc} \gamma_{i j}$ through $x_{0}$. Therefore $\ell\left(\alpha_{i j}\right) \geq \ell\left(\gamma_{i j}\right) \geq R_{i j}$. Since covering maps preserve length of curves, this implies that if $i$ is large enough no such $\alpha_{i j}$ maps to $\alpha_{g}$. Thus it is not possible to find a loop representative for $g$ in $P_{i j}$.

\section{Final remarks}

Question 3. Is it possible, in our setting, to obtain a co-final tower of regular covers $\left\{M_{j} \rightarrow M_{1}\right\}$ with positive rank gradient?

A positive answer to this would be very relevant, as it implies that Question 2 has a negative answer. However, the tower constructed in Theorem 3.1 cannot consist of normal subgroups. To see this we argue as follows: using the main theorem in [Ma] we can find a sequence $\left\{\gamma_{j}\right\}$ of hyperbolic elements, $\gamma_{j} \in G_{j}$, whose translation lengths are bounded above by 2.634 . Since there exist at most finitely many conjugacy classes of hyperbolic elements of bounded translation length in $G_{1}$, it must be that an infinite subsequence $\left\{\gamma_{j_{k}}\right\}$ lie in the same conjugacy class in $G_{1}$. Let $\gamma$ be a representative of this class and $g_{j_{k}} \in G_{1}$ be such that $\gamma_{j_{k}}=g_{j_{k}} \gamma g_{j_{k}}^{-1}$. If the tower $\left\{G_{j}\right\}$ consists of normal subgroups, then $\gamma \in G_{j_{k}}$, contradicting the fact that $\left\{G_{j_{k}}\right\}$ is co-final. These covers are actually far from being normal: the Lück Approximation Theorem ([Lu]) implies that these covers do not even satisfy a weaker condition (called Farber condition). See [Fa] for details.

Question 3 is relevant also because of the following result (see [AN]):

Theorem (Abért-Nikolov). Either the rank vs. Heegaard genus conjecture (see below) is false or Question 2 has a negative solution.

If an orientable 3-manifold $\mathrm{M}$ is closed, a Heegaard splitting of $M$ consists of two handlebodies $H_{1}$ and $H_{2}$ with their boundaries identified by some orientation preserving homeomorphism. Recall that the genus of, say, $\partial H_{1}$ gives an upper bound on the rank of $\pi_{1}(M)$. If $M$ is not closed, these decompositions are given in terms of compression bodies, again denoted by $H_{1}$ and $H_{2}$. In order to obtain useful bounds on the rank of $\pi_{1}(M)$ we restrict ourselves to those decompositions in which $H_{1}$, for instance, is a handlebody. Note that if this is the case, then the genus of $\partial H_{1}$ is again an upper bound for the rank of $\pi_{1}(M)$. Recall that the Heegaard genus of $M$ is the minimal genus of a Heegaard surface.

Another concept due to Lackenby is that of the Heegaard gradient ([La2]). Given a orientable 3-manifold $M$ and a family $\left\{M_{j}\right\}$ of finite sheeted covers, we define the Heegaard gradient of $\left\{M_{j} \rightarrow M\right\}$ by

$$
\operatorname{Hgr}\left(M,\left\{M_{j}\right\}\right)=\lim _{j \rightarrow \infty} \frac{-\chi\left(S_{j}\right)}{d_{j}}
$$


where $d_{j}$ is the degree of the cover $M_{j} \rightarrow M$ and $S_{j}$ is a minimal genus Heegaard surface for $M_{j}$.

Note that if $\operatorname{rgr}\left(M,\left\{M_{j}\right\}\right)>0$, then $\operatorname{Hgr}\left(M,\left\{M_{j}\right\}\right)>0$. The problem that is related to the work of Abért and Nikolov is that of the growth ratio between rank and Heegaard genus:

Conjecture. Let $M$ be a finite volume hyperbolic 3-manifold and $\left\{M_{i} \rightarrow M\right\}$ a family of finite sheeted covers. Then

$$
\operatorname{rgr}\left(M,\left\{M_{i}\right\}\right)>0 \text { if and only if } \operatorname{Hgr}\left(M,\left\{M_{i}\right\}\right)>0 .
$$

Our results provide examples for which this is true. In ([La2]) Lackenby showed that if $\pi_{1}(M)$ is an arithmetic lattice in $\operatorname{PSL}(2, \mathbb{C})$, then M has a co-final family of covers (namely, those arising from congruence subgroups) with positive Heegaard gradient. In [LLR] Long, Lubotzky and Reid generalize this result by proving that every finite volume hyperbolic 3-manifold has a co-final family of finite sheeted regular covers for which the Heegaard gradient is positive. These results were also motivation for this note.

We remark that very recently Tao $\mathrm{Li}([\mathrm{Li}])$ announced examples of closed finite volume hyperbolic 3-manifolds for which the rank is smaller than its Heegaard genus. Whether such examples existed was a long standing question in hyperbolic 3-manifold theory.

A natural question that arises from our results is for which other categories of finite volume hyperbolic 3-manifolds they hold. For instance:

Question 4. Is it true that given a right-angled polyhedron $P_{1}$ (not necessarily ideal) and a manifold $M_{1}$ such that $\pi_{1}\left(M_{1}\right)$ has finite index in the reflection group of $P_{1}$, then there exists a co-final tower $\left\{M_{j} \rightarrow M_{1}\right\}$ of finite sheeted covers with positive rank gradient?

In our setting the ideal vertices played an important role as they were used to find lower bounds on the rank of the fundamental groups. If the polyhedron $P_{1}$ has vertices which are not ideal then we need to find another way of estimating the rank of the associated manifolds. Ian Agol has suggested a way for doing this. We are currently working on appropriate bounds for the rank in this case and will include it in a future work.

We also remark that Lackenby ([La1]) observed that very often it is possible to find towers with positive rank gradient. The problem here is that these towers are not co-final.

Theorem 6.1. Let $M$ be a compact irreducible 3-manifold with non-empty boundary, other than an I-bundle over a disk, annulus, torus or Klein bottle. Then $\pi_{1}(M)$ has a tower of finite index subgroups with positive rank gradient 


\section{References}

[AN] M. Abért and N. Nikolov, Rank gradient, cost of groups and the rank versus Heegaard genus problem. J. Eur. Math. Soc. (JEMS) 14 (2012), 1657-1677. Zbl 1271.57046 MR 2966663

[Ag] I. Agol, Criteria for virtual fibering. J. Topol. 1 (2008), 269-284. Zbl 1148.57023 MR 2399130

[An] E. M. Andreev, On convex polyhedra in Lobačevskiǔ spaces. Mat. Sb. (N.S.) 81 (123) (1970), 445-478; English transl. Math. USSR-Sb. 10 (1970), 413-440. Zbl 0217.46801 MR 0259734

[At] C. K. Atkinson, Volume estimates for equiangular hyperbolic Coxeter polyhedra. Algebr. Geom. Topol. 9 (2009), 1225-1254. Zbl 1170.57012 MR 2519588

[De] J. DeBlois, Rank gradient of cyclic covers. Preprint 2010. http://www.pitt.edu/ jdeblois/posgrad_take2_4.pdf

[Fa] M. Farber, Geometry of growth: approximation theorems for $L^{2}$ invariants. Math. Ann. 311 (1998), 335-375. Zbl 0911.53026 MR 1625742

[Ga] D. Gaboriau, Coût des relations d'équivalence et des groupes. Invent. Math. 139 (2000), 41-98. Zbl 0939.28012 MR 1728876

[La1] M. Lackenby, Expanders, rank and graphs of groups. Israel J. Math. 146 (2005), 357-370. Zbl 1066.22008 MR 2151608

[La2] M. Lackenby, Heegaard splittings, the virtually Haken conjecture and property $(\tau)$. Invent. Math. 164 (2006), 317-359. Zbl 1110.57015 MR 2218779

[Li] T. Li, Rank and genus of 3-manifolds. J. Amer. Math. Soc. 26 (2013), 777-829. Zbl 1277.57004 MR 3037787

[Lu] W. Lück, $L^{2}$-invariants: theory and applications to geometry and K-theory. Ergeb. Math. Grenzgeb. (3) 44, Springer-Verlag, Berlin 2002. Zbl 1009.55001 MR 1926649

[LLR] D. D. Long, A. Lubotzky, and A. W. Reid, Heegaard genus and property $\tau$ for hyperbolic 3-manifolds. J. Topol. 1 (2008), 152-158. Zbl 1158.57018 MR 2365655

[LS] R. C. Lyndon and P. E. Schupp, Combinatorial group theory. Ergeb. Math. Grenzgeb. 89, Springer-Verlag, Berlin 1977. Zbl 0368.20023 MR 0577064

[Ma] J. D. Masters, Injectivity radii of hyperbolic polyhedra. Pacific J. Math. 197 (2001), 369-382. Zbl 1058.52004 MR 1815261

[Ti] J. Tits, Systèmes générateurs de groupes de congruence. C. R. Acad. Sci. Paris Sér. A 283 (1976), 693-695. Zbl 0381.14005 MR 0424966

Received September 21, 2011; revised February 15, 2012

D. Girão, Departamento de Matemática, Universidade Federal do Ceará, Av. Humberto Monte, s/n - Campus do PICI Bloco 914, Fortaleza, CE 60455-900, Brazil

E-mail: dgirao@mat.ufc.br 